\title{
THE GREENE-KRANTZ CONJECTURE IN DIMENSION TWO
}

\author{
STEVEN G. KRANTZ
}

\begin{abstract}
A proof of the Greene-Krantz conjecture on convex domains in $\mathbb{C}^{2}$ is given. Curiously, the proof technique depends on subelliptic estimates for the $\bar{\partial}$ problem.
\end{abstract}

1. Introduction. The last 35 years have seen an increase in the study of the automorphism groups of smoothly bounded domains in $\mathbb{C}^{n}$. The subject has an unusual nature because the only smoothly bounded domain with transitive automorphism group is the unit ball $B$ (see [21]). So we tend to focus our attention instead on the more general class of domains with the noncompact automorphism group. It is a classical result of Cartan [18] that such a domain $\Omega$ has the property that there is a point $P \in \Omega$ and a point $X \in \partial \Omega$ and automorphisms (i.e., biholomorphic selfmaps of $\Omega$ ) $\varphi_{j}$ such that $\varphi_{j}(P) \rightarrow X$ as $j \rightarrow \infty$. We call $X$ a boundary orbit accumulation point.

Naturally, we are interested in the geometric nature of the point $X$. It is known [5] that $X$ must in fact be a point of Levi pseudoconvexity (that is to say, the Levi form at the orbit accumulation point $X$ is positive semidefinite). But we wish to know more about the Levi geometry of $X$. With this thought in mind, the following conjecture has been formulated [5]:

Greene-Krantz conjecture. Let $\Omega$ be a smoothly bounded domain in $\mathbb{C}^{n}$. Suppose that $X \in \partial \Omega$ is a boundary orbit accumulation point for the automorphism group action in the sense that there are automorphisms $\varphi_{j}$ and a point $P \in \Omega$ such that $\varphi_{j}(P) \rightarrow X$ as

2010 AMS Mathematics subject classification. Primary 32M05, 32M12, 32M25.

Keywords and phrases. Pseudoconvex, domain, biholomorphic mapping, automorphism group, boundary orbit accumulation point.

Received by the editors on June 19, 2014, and in revised form on November 19, 2014. 
$j \rightarrow \infty$. Then $X$ is a point of finite type in the sense of Kohn/D'Angelo/Catlin.

This conjecture has been the subject of intense study for the past 20 years or more, and there are a number of interesting partial results, see for instance, $[\mathbf{7}, \mathbf{8}, \mathbf{9}, \mathbf{1 0}, \mathbf{1 1}]$. In the present paper, we prove this conjecture for smoothly bounded convex domains in complex dimension 2. It should be noted that, in complex dimension 2, the more general Kohn/D'Angelo/Catlin notion of finite type coincides with the classical notion of finite type that Kohn introduced in [12] (and that is further explicated in [13, subsection 11.5]). In what follows, we refer to the two-dimensional version of finite type as "finite type in the sense of Kohn."

2. Notation and basic ideas. We assume that the reader is familiar with complex domains and with pseudoconvexity, see [13] for background and details. When the ambient space has complex dimension 2 , there are two notions of finite type, and they are as follows.

Definition 2.1. A first order commutator of vector fields is an expression of the form

$$
[L, M] \equiv L M-M L .
$$

Note that the commutator is itself a vector field.

Inductively, an $m$ th order commutator is the commutator of an $(m-1)$ st order commutator and a vector field $L$.

Definition 2.2. A vector field of type $(1,0)$ is any linear combination of the expressions

$$
\frac{\partial}{\partial z_{1}}, \quad \frac{\partial}{\partial z_{2}},
$$

with coefficients in the ring of $C^{\infty}$ functions.

A vector field of type $(0,1)$ is any linear combination of the expressions,

$$
\frac{\partial}{\partial \bar{z}_{1}}, \quad \frac{\partial}{\partial \bar{z}_{2}},
$$

with coefficients in the ring of $C^{\infty}$ functions. 
Definition 2.3. Let $M$ be a vector field defined on the boundary of $\Omega=\left\{z \in \mathbb{C}^{2}: \rho(z)<0\right\}$. We say that $M$ is tangential if $M \rho=0$ at each point of $\partial \Omega$.

Now we define a gradation of vector fields of type $(1,0)$ which will be the basis for our definition of analytic type. Throughout this section, $\Omega=\left\{z \in \mathbb{C}^{2}: \rho(z)<0\right\}$ and $\rho$ is $C^{\infty}$ with $\nabla \rho \neq 0$ on $\partial \Omega$. If $X \in \partial \Omega$, then we may make a change of coordinates so that $\partial \rho / \partial z_{1}(X) \neq 0$. Define the vector field of type $(1,0)$,

$$
L=\frac{\partial \rho}{\partial z_{2}} \frac{\partial}{\partial z_{1}}-\frac{\partial \rho}{\partial z_{1}} \frac{\partial}{\partial z_{2}},
$$

and the vector field of type $(0,1)$,

$$
\bar{L}=\frac{\partial \rho}{\partial \bar{z}_{2}} \frac{\partial}{\partial \bar{z}_{1}}-\frac{\partial \rho}{\partial \bar{z}_{1}} \frac{\partial}{\partial \bar{z}_{2}} .
$$

Both $L$ and $\bar{L}$ are tangent to the boundary because $L \rho=0$ and $\bar{L} \rho=0$. They are both non-vanishing near $X$ by our normalization of coordinates.

The real and imaginary parts of $L$ (equivalently of $\bar{L}$ ) generate (over the ground field $\mathbb{R}$ ) the complex tangent space to $\partial \Omega$ at all points near $X$. The vector field $L$ alone generates the space of all type $(1,0)$, tangent vector fields and $\bar{L}$ alone generates the space of all tangent vector fields of type $(0,1)$.

Definition 2.4. Let $\mathcal{L}_{1}$ denote the module, over the ring of $C^{\infty}$ functions, generated by $L$ and $\bar{L}$. Inductively, $\mathcal{L}_{\mu}$ denotes the module generated by $\mathcal{L}_{\mu-1}$ and all commutators of the form $[F, G]$ where $F \in \mathcal{L}_{1}$ and $G \in \mathcal{L}_{\mu-1}$.

Clearly, $\mathcal{L}_{1} \subseteq \mathcal{L}_{2} \subseteq \cdots$. Each $\mathcal{L}_{\mu}$ is closed under conjugation. It is not generally the case that $\cup_{\mu} \mathcal{L}_{\mu}$ is the entire three-dimensional tangent space at each point of the boundary. A counterexample is provided by

$$
\Omega=\left\{z \in \mathbb{C}^{2}:\left|z_{1}\right|^{2}+2 e^{-1 /\left|z_{2}\right|^{2}}<1\right\}
$$

and the point $X=(1,0)$. 
Definition 2.5. Let $\Omega=\{\rho<0\}$ be a smoothly bounded domain in $\mathbb{C}^{2}$, and let $X \in \partial \Omega$. We say that $\partial \Omega$ is of finite analytic type $m$ at $X$ if $\langle\partial \rho(X), F(X)\rangle=0$ for all $F \in \mathcal{L}_{m-1}$ while $\langle\partial \rho(X), \gamma(X)\rangle \neq 0$ for some $\gamma \in \mathcal{L}_{m}$. In this circumstance we call $X$ a point of analytic type $m$.

Now we turn to a precise definition of finite geometric type. Let $D$ denote the unit disc in the complex plane. If $X$ is a point in the boundary of a smoothly bounded domain, then we say that an analytic disc $\phi: D \rightarrow \mathbb{C}^{2}$ is a non-singular disc tangent to $\partial \Omega$ at $X$ if $\phi(0)=X$, $\phi^{\prime}(0) \neq 0$ and $(\rho \circ \phi)^{\prime}(0)=0$.

Definition 2.6. Let $\Omega=\{\rho<0\}$ be a smoothly bounded domain and $X \in \partial \Omega$. Let $m$ be a non-negative integer. We say that $\partial \Omega$ is of finite geometric type $m$ at $X$ if the following condition holds: there is a non-singular disc $\phi$ tangent to $\partial \Omega$ at $X$ such that, for small $\zeta$,

$$
|\rho \circ \phi(\zeta)| \leq C|\zeta|^{m} \text {. }
$$

But, there is no non-singular disc $\psi$ tangent to $\partial \Omega$ at $X$ such that, for small $\zeta$

$$
|\rho \circ \phi(\zeta)| \leq C|\zeta|^{(m+1)} .
$$

In this circumstance we call $X$ a point of finite geometric type $m$.

A basic result about finite type in dimension 2 is the following theorem (see $[\mathbf{1}, \mathbf{1 2}])$.

Theorem 2.7. Let $\Omega=\{\rho<0\} \subseteq \mathbb{C}^{2}$ be smoothly bounded and $X \in \partial \Omega$. The point $X$ is of finite geometric type $m \geq 2$ if and only if it is of finite analytic type $m$.

See [13, subsection 11.5] for a simplified version of the proof.

In complex dimension 2, we shall refer to either geometric or analytic finite type as "finite type in the sense of Kohn."

We shall make use of a new function space in what follows. 
Definition 2.8. If $0<\alpha<1$, then we say that a function $f$ lies in the $p$-degree $K$-space of order $\alpha$ if

$$
\|f(\cdot+h)-f(\cdot)\|_{L^{p}} \leq\left(C+\|f\|_{L^{p}}\right) \cdot\|h\|^{\alpha} .
$$

The norm on such a $K$-space is

$$
\|f\|_{K} \equiv \sup _{h \neq 0} \frac{\|f(\cdot+h)-f(\cdot)\|_{L^{p}}}{|h|^{\alpha}}+\|f\|_{L^{p}} .
$$

This space of functions is analogous to the traditional Lipschitz spaces and also bears some similarities to Besov spaces. But, it is not one of the familiar function spaces. It is discussed explicitly in [22, page 45]. It is straightforward to see that the 2-degree $K$-space of order $\alpha$ is comparable to the Sobolev space of order $\alpha$.

Now, let us say a few words about subelliptic estimates. A partial differential operator $\mathcal{L}$ of order $k$ is said to satisfy elliptic estimates if, whenever $\mathcal{L} u=f$ and $f$ lies in the Sobolev space $W^{s}$, then $u$ lies in the Sobolev space $W^{s+k}$. The operator is said to satisfy subelliptic estimates if the index $s+k$ in the conclusion is replaced by $s+k^{\prime}$ for some $0<k^{\prime}<k$. The $\bar{\partial}$ operator on a strongly pseudoconvex domain, and more generally on a finite type domain, is known to satisfy a subelliptic (but definitely not an elliptic) estimate, see $[\mathbf{2}, \mathbf{3}, \mathbf{4}, \mathbf{1 5}]$ for the details. It is also possible to express the subellipticity condition in terms of Lipschitz, Triebel-Lizorkin or $K$-spaces rather than Sobolev spaces. We leave the details for the interested reader.

3. The main argument. The result that we shall actually prove in this paper is the following.

Theorem 3.1. Let $\Omega$ be a smoothly bounded, convex domain in $\mathbb{C}^{2}$. Suppose that $X \in \partial \Omega$ is a boundary orbit accumulation point for the automorphism group action in the sense that there are automorphisms $\varphi_{j}$ and a point $P \in \Omega$ such that $\varphi_{j}(P) \rightarrow X$ as $j \rightarrow \infty$. Then $X$ is a point of finite type in the sense of Kohn.

Certainly, it should be mentioned that a result about the GreeneKrantz conjecture in dimension 2 is proved in [16]. Related results appear in $[\mathbf{1 1}, \mathbf{1 7}]$. 
Now fix a smoothly bounded domain $\Omega \subseteq \mathbb{C}^{2}$. Assume that $P \in \Omega$ and $X \in \partial \Omega$ and that there are automorphisms $\varphi_{j}$ of $\Omega$ such that $\varphi_{j}(P) \rightarrow X$ as $j \rightarrow \infty$. Note that, because the domain $\Omega$ is smoothly bounded and pseudoconvex, it is complete in the Bergman metric, see [19].

Next, consider a small Bergman metric ball $\beta$ centered at $P$. Choose $j_{1}$ so that $\beta_{1} \equiv \varphi_{j_{1}}(\beta)$ is disjoint from $\beta$, and so that the Euclidean distance of $\beta_{1}$ to the boundary is between $2^{-1} / 2$ and $2 \cdot 2^{-1}$. Now choose $j_{2}$ so that $\beta_{2} \equiv \varphi_{j_{2}}(\beta)$ is disjoint from $\beta$ and $\varphi_{1}(\beta)$ and so that the Euclidean distance of $\beta_{2}$ to the boundary is between $2^{-2} / 2$ and $2 \cdot 2^{-2}$. Iterate this procedure.

Finally, fix a $\bar{\partial}$-closed $(0,1)$ form $\psi$ with $C_{c}^{\infty}$ coefficients that is supported in $\beta$. Define $\psi_{\ell}=\left(\varphi_{j_{\ell}}^{-1}\right)^{*} \psi$. Thus, $\psi_{\ell}$ is a $\bar{\partial}$-closed $(0,1)$ form with $C_{c}^{\infty}$ coefficients supported on $\beta_{\ell}$. Because of the size of the sup norm of the derivative of $\varphi_{j_{\ell}}, \psi_{\ell}$ has supremum norm about $2^{-\ell}$. This means that the sum of the $\psi_{\ell}$ will have an $L^{2}$ or Sobolev norm which converges.

If we write $\psi_{\ell}=\psi_{\ell}^{1} d \bar{z}_{1}+\psi_{\ell}^{2} \bar{z}_{2}$, then we may note that the equation $\bar{\partial} u_{\ell}=\psi_{\ell}$ can be solved by one of the simple equations:

$$
u_{\ell}^{1}\left(z_{1}, z_{2}\right)=\iint_{\zeta \in \mathbb{C}} \frac{\psi_{\ell}^{1}\left(\zeta, z_{2}\right)}{\zeta-z_{1}} d A(\zeta)
$$

or

$$
u_{\ell}^{2}\left(z_{1}, z_{2}\right)=\iint_{\zeta \in \mathbb{C}} \frac{\psi_{\ell}^{2}\left(z_{1}, \zeta\right)}{\zeta-z_{2}} d A(\zeta)
$$

see $\left[\mathbf{1 3}\right.$, subsection 1.1]. It turns out that $u_{\ell}^{1}=u_{\ell}^{2}$.

It follows from standard results on fractional integration (see [20, pages 117-121]) that, if $\psi_{\ell}$ is in some Sobolev class $W^{s}$, then $u_{\ell}^{m}$ will be in a smoother Sobolev class $W^{s^{\prime}}$, with $s^{\prime}>s$, in the $m$ th variable, $m=1,2$.

And now a simple argument with the triangle inequality shows that $u_{\ell}^{m}$ lies in $W^{s^{\prime \prime}}$ as a function of both variables for some $s^{\prime} \geq s^{\prime \prime}>s$, $m=1,2$. So we see that the $\bar{\partial}$ problem satisfies a subelliptic estimate on $\psi_{\ell}$. 
But, in fact, due to the intervention of the automorphisms $\varphi_{\ell}$, the $\bar{\partial}$ problem satisfies the very same subelliptic estimate for each $\psi_{\ell}$. (By this we mean a subelliptic estimate with the same norms and the same constant.) As a result, the $\bar{\partial}$ problem satisfies a subelliptic estimate on the form

$$
\psi \equiv \sum_{\ell} \psi_{\ell}
$$

Therefore, it is definitely not the case that the $\bar{\partial}$-closed $(0,1)$ forms with $C_{c}^{\infty}$ coefficients are dense in any space of forms with Sobolev coefficients. But we shall be able to argue that they are dense in a space containing certain forms that we care about. (See also the endnote.)

We have the following lemma.

Lemma 3.2. If the boundary orbit accumulation point $X$ is of infinite type, then, for each $\epsilon>0$, there is a $\bar{\partial}$-closed $(0,1)$ form $f$ on $\Omega$ with $L^{2}$ coefficients so that the equation $\bar{\partial} u=f$ does not have any solution in the $K$-space of order $\epsilon>0$.

After the proof of the lemma we shall relate this construction to the main result. The idea for the proof goes back to an old result of Kerzman [6] and is reasonably well known, see also [13, subsection 10.3].

Proof of Lemma 3.2. We sketch the idea here.

We may assume that $X=(1,0) \in \partial \Omega$ and that the complex normal direction at $X$ is $\langle 1,0\rangle$. We write $\nu_{X}=\langle 1,0\rangle$. With these normalizations, we define

$$
f=\frac{d \bar{z}_{2}}{\log \left(1-z_{1}\right)} .
$$

By the convexity of $\Omega$, it is clear that the principal branch of the logarithm is well defined and that $f$ has bounded coefficients.

Now any solution of the equation $\bar{\partial} u=f$ will have the form,

$$
u(z)=\frac{\bar{z}_{2}}{\log \left(1-z_{1}\right)}+h\left(z_{1}, z_{2}\right)
$$

where $h$ is some holomorphic function on $\Omega$. 
Since $X$ is a point of infinite type, then we know that, for any positive integer $m$, there is a nonsingular complex curve $\lambda_{m}: D \rightarrow \mathbb{C}^{2}$ that is tangent to order at least $2 m$ with $\partial \Omega$ at $X$. Then, for $\delta>0$ small, the analytic disc

$$
\left\{\lambda_{m}(\zeta)-\delta \nu_{X}:|\zeta|<C \delta^{1 /(2 m)}, \zeta \in D\right\}
$$

lies in $\Omega$ (see [14, pages 171-172] for the elementary calculations needed to justify this assertion; this is just interpreting the definition of finite type). Thus,

$$
\theta_{\delta}: t \longmapsto \lambda_{m}\left(C \delta^{1 /(2 m)} e^{i t}\right)-\delta \nu_{X}, \quad 0 \leq t<2 \pi,
$$

describes the boundary of an analytic disc in $\Omega$.

We may suppose that $(1,0) \in \partial \Omega$ is our point of infinite type (arranged by a simple change of coordinates). Take $W$ to be a small neighborhood of $(1,0)$. We may assume that $W$ is an Euclidean ball, and that it lies in a tubular neighborhood of $\partial \Omega$. Pick $\delta>0$ small enough so the $\delta^{1 /(2 m)}$ is much less than the radius of $W$, and define

$$
\widetilde{\Omega}=(W \cap \Omega) \bigcap\left\{z \in \Omega: \delta^{1 / m}>\operatorname{dist}(z, \partial \Omega)>\delta\right\} .
$$

We examine the complex line integral,

$$
F(\delta, \zeta)=\oint_{\theta_{\delta}} u\left(\zeta_{1}-2 \delta, \zeta_{2}+z_{2}\right)-u\left(\zeta_{1}-\delta, \zeta_{2}+z_{2}\right) d z_{2},
$$

for $\zeta \in \widetilde{\Omega}$. We note that the curves

$$
t \longmapsto-\delta \nu_{X}+\lambda_{m}\left(C^{\prime} \delta^{1 /(2 m)} e^{i t}\right)
$$

and

$$
t \longmapsto-2 \delta \nu_{X}+\lambda_{m}\left(C^{\prime} \delta^{1 /(2 m)} e^{i t}\right),
$$

where $C^{\prime}$ is a small positive constant depending on the geometry of $\partial \Omega$ near $(1,0)$, both lie in $\Omega$ precisely because $X$ is a point of infinite type (more precisely, a point of type at least $2 m$ ); again, see [14, pages $171-172]$. 
Seeking a contradiction, if $u$ satisfies a $K$-space condition of order $\epsilon$, then we may straightforwardly estimate that

$$
\begin{aligned}
\|F(\delta, \zeta)\|_{L^{2}(\zeta)} \leq & \int_{\tilde{\Omega}} \mid \oint_{\theta_{\delta}} u\left(\zeta_{1}-2 \delta, \zeta_{2}+z_{2}\right) \\
& -\left.u\left(\zeta_{1}-\delta, \zeta_{2}+z_{2}\right) d z_{2}\right|^{2} d V(\zeta)^{1 / 2} \\
& \leq \int_{\theta_{\delta}} \int_{\tilde{\Omega}} \mid u\left(\zeta_{1}-2 \delta, \zeta_{2}+z_{2}\right) \\
& \leq C \cdot \int_{\theta_{\delta}} \delta^{\epsilon} d\left|z_{2}\right| \approx C \cdot \delta^{\epsilon+1 /(2 m)} .
\end{aligned}
$$

On the other hand,

$$
\begin{aligned}
F(\delta, \zeta) & =\int_{\theta_{\delta}} \frac{\bar{z}_{2}+\bar{\zeta}_{2}}{\log \left(1-\zeta_{1}+2 \delta\right)}-\frac{\bar{z}_{2}+\bar{\zeta}_{2}}{\log \left(1-\zeta_{1}+\delta\right)} d z_{2} \\
& =\frac{\delta^{2 /(2 m)}}{\log \left(1-\zeta_{1}+2 \delta\right)}-\frac{\delta^{2 /(2 m)}}{\log \left(1-\zeta_{1}+\delta\right)}
\end{aligned}
$$

Therefore,

$$
|F(\delta, \zeta)| \approx C \cdot \frac{\delta^{1 / m}}{\log ^{2}(\delta)}
$$

As a result,

$$
\int_{\tilde{\Omega}}|F(\delta, \zeta)|^{2} d V(\zeta)^{1 / 2} \approx \frac{\delta^{1 / m}}{\log ^{2}(\delta)} \cdot \delta^{1 / m}
$$

Comparing our two estimates, we find that

$$
\frac{\delta^{1 / m} \cdot \delta^{1 / m}}{\log ^{2}(\delta)} \leq C \cdot \delta^{\epsilon+1 /(2 m)}
$$

or

$$
\frac{\delta^{3 /(2 m)}}{\log ^{2}(\delta)} \leq C \cdot \delta^{\epsilon}
$$

This is false for $\delta \rightarrow 0$ when $m \in \mathbb{N}$ is large enough. 
Lemma 3.2 tells us that, in the $K$-space topology, the $\bar{\partial}$ problem does not satisfy a subelliptic estimate. But it is not difficult to see that the form

$$
f(z)=\frac{d \bar{z}_{2}}{\log \left(1-z_{1}\right)}
$$

is the limit of forms with compact support. ${ }^{1}$ Let $\rho_{2}$ be a $C_{c}^{\infty}$ function that approximates $1 / \log \left(1-z_{1}\right)$ in the $L^{2}$ topology. Now the formula

$$
v\left(z_{1}, z_{2}\right)=\iint \frac{\rho_{2}\left(z_{1}, \zeta\right)}{\zeta-z_{2}} d \zeta
$$

satisfies

$$
\frac{\partial}{\partial \bar{z}_{2}} v=\rho_{2} .
$$

Notice that $v$ has compact support by classical arguments presented in [13, Chapter 0].

Note that (see [13, subsection 1.1]) $v \in C_{c}^{\infty}(\Omega)$. Hence,

$$
\rho_{1}(z) \equiv \frac{\partial}{\partial \bar{z}_{1}} v
$$

will give a form

$$
R=\rho_{1} d \bar{z}_{1}+\rho_{2} d \bar{z}_{2},
$$

that is $\bar{\partial}$-closed with $C_{c}^{\infty}$ coefficients. And, of course, $R$ will approximate $f$ in the $L^{2}$ topology.

Additionally, this approximation implies that the problem $\bar{\partial} u=f$, with $f$ as in Lemma 3.2, satisfies a subelliptic estimate in the Sobolev topology. But this implies that it satisfies a subelliptic estimate in the $K$-space topology and we have established in the lemma that that is impossible.

We have proved that the boundary orbit accumulation point $X$ cannot be of infinite type.

Remark 3.3. It is worth noting that the construction presented here, of the ball $\beta$ and subsequent target balls $\varphi_{j_{1}}(\beta), \varphi_{j_{2}}(\beta)$, etc., does not work when the automorphism group is compact. For, when the automorphism group is compact, then these balls will no longer be pairwise disjoint. Also, the norms of the $\left(\varphi_{j_{k}}^{-1}\right)^{*} \psi$ will no longer 
vanish rapidly so that the series which is obtained by adding the forms supported on the different balls will no longer converge.

4. Concluding remarks. In this paper, we certainly have not proved the full Greene-Krantz conjecture. But, we have proved a notable and interesting special case.

There is certainly interest in developing techniques for attacking the full conjecture, and we intend to attack that problem in future papers.

Acknowledgments. It is a pleasure to thank Harold Boas and Emil Straube for useful comments and suggestions.

\section{ENDNOTES}

And notice that, if $\psi_{0}$ is a $\bar{\partial}$-closed $(0,1)$ form with $C_{c}^{\infty}$ coefficients on $\beta$, then we can consider the form $\psi$ on the union of $\beta, \varphi_{j_{1}}(\beta)$, etc., as described above, and we can also consider the "shifted" form $\tau$ given by $\left(\varphi_{j_{1}}^{-1}\right)^{*} \psi$ on $\varphi_{j_{1}}(\beta),\left(\varphi_{j_{2}}^{-1}\right)^{*} \psi$ on $\varphi_{j_{2}}(\beta)$ (with intervening automorphism $\left.\varphi_{j_{2}} \circ \varphi_{j_{1}}^{-1}\right)$, and so forth. Then the difference of these two forms is a $C_{c}^{\infty}$ form supported on $\beta$ alone. So, our arguments and estimates also apply to forms that have compact support and are smooth.

\section{REFERENCES}

1. T. Bloom and I. Graham, A geometric characterization of points of type $m$ on real submanifolds of $\mathbb{C}^{n}$, J. Differential Geom. 12 (1977), 171-182.

2. D. Catlin, Necessary conditions for subellipticity of the $\bar{\partial}$-Neumann problem, Annals Math. 117 (1983), 147-172.

3. Subelliptic estimates for the $\bar{\partial}-$ Neumann problem, Annals Math. 126 (1987), 131-192.

4. G.B. Folland and J.J. Kohn, The Neumann problem for the Cauchy-Riemann complex, Princeton University Press, Princeton, NJ, 1972.

5. R.E. Greene and S.G. Krantz, Invariants of Bergman geometry and results concerning the automorphism groups of domains in $\mathbb{C}^{n}$, Sem. Conf. 8, EditEl, Rende, 1991, 107-136.

6. N. Kerzman, Hölder and $L^{p}$ estimates for solutions of $\bar{\partial} u=f$ on strongly pseudoconvex domains, Comm. Pure Appl. Math. 24 (1971), 301-380.

7. K.-T. Kim, Domains in $\mathbb{C}^{n}$ with a piecewise Levi flat boundary which possess a noncompact automorphism group, Math. Ann. 292 (1992), 575-586. 
8. K.-T. Kim and S.G. Krantz, Complex scaling and domains with non-compact automorphism group, Illinois J. Math. 45 (2001), 1273-1299.

9. automorphism group, J. Math. Anal. Appl. 281 (2003), 417-424.

10. Complex scaling and geometric analysis of several variables, Bull. Korean Math. Soc. 45 (2008), 523-561.

11. S.-Y. Kim, Domains with hyperbolic orbit accumulation boundary points, J. Geom. Anal. 22 (2012), 90-106.

12. J.J. Kohn, Boundary behavior of $\bar{\partial}$ on weakly pseudoconvex manifolds of dimension two, J. Differential Geom. 6 (1972), 523-542.

13. S.G. Krantz, Function theory of several complex variables, 2nd. edition, American Mathematical Society, Providence, RI, 2001.

14. Characterizations of various domains of holomorphy via $\bar{\partial}$ estimates and applications to a problem of Kohn, Illinois J. Math. 23 (1979), 267285.

15. Partial differential equations and complex analysis, CRC Press, Boca Raton, FL, 1992.

16. M. Landucci, The automorphism group of domains with boundary points of infinite type, Illinois J. Math. 48 (2004), 875-885.

17. M. Landucci and G. Patrizio, Unbounded domains in $\mathbb{C}^{2}$ with non-compact automorphism group, Results Math. 42 (2002), 300-307.

18. R. Narasimhan, Several complex variables, University of Chicago Press, Chicago, 1971.

19. T. Ohsawa, A remark on the completeness of the Bergman metric, Proc. Japan Acad. Math. Sci. 57 (1981), 238-240.

20. E.M. Stein, Singular integrals and differentiability properties of functions, Princeton University Press, Princeton, NJ, 1970.

21. B. Wong, Characterizations of the ball in $\mathbb{C}^{n}$ by its automorphism group, Invent. Math. 41 (1977), 253-257.

22. A. Zygmund, Trigonometric series, Cambridge, University Press, Cambridge, 1968.

Department of Mathematics, Washington University in St. Louis, St. Louis, MO 63130

Email address: sk@math.wustl.edu 